\title{
Group efficiency analysis in decision processes: a data envelopment analysis approach
}

\author{
Mohsen Hekmatnia $^{1}$, Alireza Amirteimoori ${ }^{1, *}$, Sohrab Kordrostami ${ }^{2}$ \\ ${ }^{1}$ Department of Applied Mathematics, Islamic Azad University \\ Rasht branch, Rasht, Iran \\ E-mail: 〈\{hekmatnia, ateimoori\}@iaurasht.ac.ir〉 \\ 2 Department of Applied Mathematics, Islamic Azad University \\ Lahijan branch, Lahijan, Iran \\ E-mail: 〈sohrabkordrostami@gmail.com〉
}

\begin{abstract}
Data envelopment analysis (DEA) is a powerful mathematical programming methodology for evaluating the relative efficiency of decision-making units (DMUs) with multiple outputs and multiple inputs. In the classic DEA, it has been implicitly assumed that all DMUs perform in a unique technology set and the traditional DEA cannot measure the relative performances of DMUs with dissimilar classes. In other words, if we have different groups of DMUs, the traditional DEA models cannot be applied to evaluate such cases. In this paper, it has been assumed that the DMUs do business in different groups. We are interested to evaluate the members of the groups. The main aim of this paper is proposing a DEA-based methodology to estimate the technical efficiency of DMUs along with different groups with different technologies. The proposed method is illustrated by an empirical example on banking industry.
\end{abstract}

Keywords: banking industry, data envelopment analysis, group efficiency, input, output

Received: July 6, 2018; accepted: January 23, 2019; available online: July 4, 2019

DOI: $10.17535 /$ crorr.2019.0008

\section{Introduction}

Discussion on how to evaluate the relative performances of the firms is very important and many researchers in the field of performance management and measurement have made substantial contribution in the last four decades. Following the pioneering work of [7], a body of literature has been developed to analyze the relative performances of homogeneous firms.

One of the most frequently used and representative methods of performance analysis is data envelopment analysis (DEA). In the last three decades, DEA, initiated by [5] and developed by [2] has been proven as an excellent data-oriented efficiency analysis method for comparing decision making units (DMUs) with multiple outputs and multiple inputs. DEA is a commonly used tool in performance evaluation of firms in the sense that DEA models do not estimate the exact production frontier and using the observed inputs/outputs data, an empirical production frontier is constructed to analysis of the firm's performances. Recently, many applications of DEA have been reported in the literature. For more information about the applications of DEA, see [6].

In traditional DEA, we have a set of DMUs that do business in the same technological environment and the DMUs use same inputs to produce same outputs. In real application in

${ }^{*}$ Corresponding author.

http://www.hdoi.hr/crorr-journal

(C)2019 Croatian Operational Research Society 
performance analysis of the firms, despite of the fact that the DMUs are homogeneous, they may perform in different groups with different technologies. So, there is a need to provide modified models and methods to evaluate the DMUs in such a cases. One way to evaluate the relative performances of the DMUs in different groups with same technology is classifying DMUs into different categories. In DEA, categorical DMUs are classified into controllable and non-controllable by decision maker. Then, DEA tries to solve this problem by a special algorithm. As mentioned earlier, the traditional DEA models such as "DEA with categorical DMUs" implicitly consider a same technology for all DMUs and in a special case, they didn't allow DMUs with different categories.

Discussion on the performance analysis with technological differences is an important subject that has been studied by DEA researchers. In all studies, making an envelope to the DMUs in the groups is proposed as a solution to this problem. In the DEA literature, such envelopment is referred to as meta-frontier.

The meta-frontier is defined as a common boundary that envelopes every group of DMUs. The idea of meta-frontier was first introduced by $[9,10,15]$ have used the meta-frontier concept in their studies. O'Donnell [11] proposed a meta-frontier framework to study the firm level efficiencies. The meta-frontier constructed in all of the existing approaches is not a convex function. This paper has a different look at the group performance analysis. The method proposed in this paper is different from those that have been studied in previous works. As we know, empirical production frontier is our estimation related to the transformation of inputs to outputs and DEA overestimates the efficiencies of the DMUs, so, we need to evaluate the DMUs in a more solid condition. We assume that we have case in which there are $K$ different groups and in each group a set of DMUs are doing business separately. The groups that the DMUs belong to are almost same and in this sense, some DMUs may change their group to tryout their performances. So, the DMUs can be transferred from one group to another one. To evaluate a specific DMU, a mixed integer linear programming problem is proposed and using the proposed model, we implicitly evaluate the DMU under consideration in each of the groups and the most favorable group is chosen to each DMU as the reference group.

The rest of this paper is organized as follows: In the next section (Section 2), performance analysis in the groups is given. Analyzing the problem of transferring a DMU from its current group to a specific group is given in Section 3. An application to a major Iranian bank is given in Section 4. Conclusions appear in Section 5.

\section{Performance measurement in groups}

\subsection{Meta-technology}

Suppose there are $n$ DMUs to be evaluated in K different groups and the $k$-th group $G_{k}$ consists of $D M U_{j 1}, D M U_{j 2}, \ldots, D M U_{j k}$. Each $D M U_{j k}$ uses $m_{k}$ inputs $\left(x_{1 j k}, x_{2 j k}, \ldots, x_{m j k}\right) \geq 0$ to produce $s$ outputs $\left(y_{1 j k}, y_{2 j k}, \ldots, y_{s j k}\right) \geq 0$. An important point to be noted is that although each $D M U_{j}$ faces to a different technology, but the groups are homogeneous in the sense that the DMUs in two different groups uses same inputs to generate same outputs and due to this similarity, in case of need, in order to improve their performances, they are allowed to be transferred from one group to another one. Battese et al. [3] argued that since a technology set is our knowledge related to the transformation of inputs to outputs, it is possible to conceptualize the existence of a meta-technology. O'Donnell et al. [11] have used the concept of meta-frontier to compute the technical efficiency of the DMUs that may be classified in to different groups with same technology.

Suppose $T_{G 1}, T_{G 2}, \ldots, T_{G k}$ are $K$ different technologies with $K$ different natures. The metatechnology set $T_{M}$ in our analysis is defined as follows: 


$$
T_{M}=\left\{(x, y): \exists k \in\{1,2, \ldots, K\}:(x, y) \in T_{G k}\right\}
$$

Indeed, $T_{M}$ consists of all production possibilities $(x, y)$ that belong to one of the technologies $T_{G k}, k=1,2, \ldots, K$.

Consider the $k$-th technology set as follows:

$$
T_{G k}=\left\{(x, y): x \geq \sum_{j \in j_{k}} \lambda_{j}^{k} x_{j}^{k}, y \leq \sum_{j \in j_{k}} \lambda_{j}^{k} y_{j}^{k}, \quad \lambda^{k}=\left(\lambda_{1}^{k}, \ldots, \lambda_{j k}^{k}\right) \in \Lambda_{k}\right\}
$$

in which $\Lambda_{k}$ is used to clarify the nature of $T_{G k}$. Based on the definition of $T_{M}$ an algebraic representation of the production set $T_{M}$ is given as follows:

$$
\begin{gathered}
T_{M}=\left\{(x, y): x \geq \sum_{k=1}^{K} \mu^{k}\left(\sum_{j \in j_{k}} \lambda_{j}^{k} x_{j}^{k}\right), y \leq \sum_{k=1}^{K} \mu^{k}\left(\sum_{j \in j_{k}} \lambda_{j}^{k} y_{j}^{k}\right), \sum_{k=1}^{K} \mu^{k}=1,\right. \\
\left.\lambda^{k}=\left(\lambda_{1}^{k}, \ldots, \lambda_{j k}^{k}\right) \in \Lambda_{k}, \mu^{k} \in\{0,1\}\right\}
\end{gathered}
$$

As we stated before, the meta-technology $T_{M}$ is the union of the technology sets, $T_{G k}, k=$ $1,2, \ldots, K$. The boundary points of the metatechnology $T_{M}$ is the metafrontier and this frontier plays the main role in our analysis. A DMU under evaluation would be assessed to the part of this frontier to maximize its performance.

\subsection{Technical efficiency measurement}

Now, we come to evaluate the relative efficiency of a specific $D M U_{o}$ To describe the DEA efficiency measurement of the $D M U_{o}$, we use the directional distance function as follows:

$$
D F\left(x_{o}, y_{o}\right)=\operatorname{Max}\left\{1 \delta+1 \rho:\left(x_{o}-\delta d^{x}, y_{o}+\rho d^{y}\right) \in T_{M}, 0 \leq \delta \leq 1, \rho \geq 0\right\}
$$

in which $\delta=\left(\delta_{1}, \delta_{2}, \ldots, \delta_{m}\right), \rho=\left(\rho_{1}, \rho_{2}, \ldots, \rho_{s}\right)$ and $1=(1,1, \ldots, 1)$ is used to summation. $d^{x}=\left(d_{1}^{x}, d_{2}^{x}, \ldots, d_{m}^{x}\right)$ and $d^{x}=\left(d_{1}^{y}, d_{2}^{y}, \ldots, d_{s}^{y}\right)$ are user-defined values that are used to show the directional distance. Based on the definition of the meta-technology $T_{M}$ model in eq.(4) becomes as the following form:

$$
\begin{array}{ll}
e_{o}^{*}=\operatorname{Max} \sum_{i=1}^{m} \delta_{i}^{o}+\sum_{r=1}^{s} \rho_{r}^{0} & \\
\sum_{k=1}^{K} \mu^{k}\left(\sum_{j \in j_{k}} \lambda_{j}^{k} x_{i j}^{k}\right) \leq x-\delta_{i}^{o} d_{i o}^{x}, & i=1,2, \ldots, m \quad k=1,2, \ldots, K \\
\sum_{k=1}^{K} \mu^{k}\left(\sum_{j \in j_{k}} \lambda_{j}^{k} y_{r j}^{k}\right) \geq y+\rho_{r}^{o} d_{r o}^{y}, & r=1,2, \ldots, s \quad k=1,2, \ldots, K \\
\sum_{k=1}^{K} \mu^{k}=1, & \\
\lambda^{k}=\left(\lambda_{1}^{k}, \ldots, \lambda_{j k}^{k}\right) \in \Lambda_{k}, & k=1,2, \ldots, K \\
0 \leq \delta_{i} \geq, & i=1,2, \ldots, m \\
\rho_{r} \geq 0, & r=1,2, \ldots, s
\end{array}
$$


In given model, each $D M U_{o}$ is evaluated with respect to each of the groups and its efficiency score is obtained. When we maximize $\delta_{i}^{o}+\rho_{r}^{o}$, we try to evaluate the $D M U_{o}$ to those frontier that $D M U_{o}$ has maximum distance to the frontier. This shows the inefficiency of $D M U_{o}$. The binary variable $\mu_{k}$ is used to show if $G_{k}$ is considered as the reference group to $D M U_{o}$ or not. $\mu_{k}=1$ shows that the $k$-th group is the reference group to $D M U_{o}$ and hence the reference set of $D M U_{o}$ is selected among DMUs in $G_{k}$. It should be noted that if $E_{o}$ be the reference set of $D M U_{o}$, all members of $E_{o}$ belong to a same group $G$ and this is impossible to $E_{o}$ to have members from two different $G_{k}$.

Model given by eq.(5) is a mixed integer nonlinear programming problem. In order to linearize the above model, we use the following changes of variables:

$$
\bar{\lambda}_{j}^{k}=\mu^{k} \lambda_{j}^{k}, \quad k=1,2, \ldots, K \quad j=1,2, \ldots, j_{k}
$$

If $\mu_{k}=0$ we must have $\bar{\lambda}_{j}^{k}=0$ and when $\mu_{k}=1$ we must have $\bar{\lambda}_{j}^{k}=\lambda_{j}^{k}$ for all $j=1,2, \ldots, j_{k}$. Supose $M$ be a large positive number and consider the following constraints:

$$
0 \leq \bar{\lambda}_{j k}^{k} \leq \mu^{k} M, \quad k=1,2, \ldots, K \quad \forall j \in j_{k}
$$

Clearly $\mu^{k}=0$ forces $\bar{\lambda}_{j}^{k}=0$ for all $j=1,2, \ldots, j_{k}$ and if $\mu_{k}=1$ then $\bar{\lambda}_{j}^{k} \geq 0$ for all $j=1,2, \ldots, j_{k}$, and this is what we want. With these changes of variables, model in eq.(5) becomes of the following form:

$$
\begin{array}{ll}
e_{o}^{*}=\operatorname{Max} \sum_{i=1}^{m} \delta_{i}^{o}+\sum_{r=1}^{s} \rho_{r}^{0} & \\
\sum_{k=1}^{K} \sum_{j \in j_{k}} \bar{\lambda}_{j k}^{k} x_{i j}^{k} \leq x-\delta_{i}^{o} d_{i o}^{x}, & i=1,2, \ldots, m \quad k=1,2, \ldots, K \\
\sum_{k=1}^{K} \sum_{j \in j_{k}} \bar{\lambda}_{j k}^{k} y_{r j}^{k} \geq y+\rho_{r}^{o} d_{r o}^{y}, & r=1,2, \ldots, s \quad k=1,2, \ldots, K \\
\sum_{k=1}^{K} \mu^{k}=1, & \\
0 \leq \bar{\lambda}_{j k}^{k} \geq \mu^{k} M, & k=1,2, \ldots, K \quad j \in j_{k} \\
\bar{\lambda}^{k}=\left(\bar{\lambda}_{1}^{k}, \ldots, \bar{\lambda}_{j k}^{k}\right) \in \bar{\Lambda}_{k}, & k=1,2, \ldots, K \\
\mu^{k} \in\{0,1\}, & k=1,2, \ldots, K \\
0 \leq \delta_{i} \geq, & i=1,2, \ldots, m \\
\rho_{r} \geq 0, & r=1,2, \ldots, s
\end{array}
$$

Note that the form of $\bar{\Lambda}_{k}$ depends of the form of $\Lambda_{k}$ and $\mu^{k}$. Model (8) is a mixed integer linear programming problem. It is easy to show the feasibility and the boundedness of this problem. In model (6), $D M U_{o}$ is assessed to all of the groups and in the objective function, we look for the maximum reduction in the inputs and maximum expansion in the outputs, while remaining in some technology set $T_{G i}$.

Although the individual technologies $T_{G 1}, T_{G 2}, \ldots, T_{G k}$ are convex sets, we should not expect the new meta-technology be a convex set. Despite of this, the DMU under evaluation, is assessed to the part of the meta-technology that is a convex function and the corresponded technology is a convex set. This guaranties that the local optimal solution obtained from model (8) is a global optimal solution. This is the proof of the following theorem. 
Theorem 1. Each local optimal solution to the mathematical programming problem (8) is a global optimal solution to this problem.

Definition 1. $D M U_{o}$ is said to be full efficient if and only if the objective function value of model (8) is zero and all slack variables are zero.

Theorem 2. The new score $e_{o}^{*}$ of $D M U_{o}$ in the whole group is greater than or equal to its old score in its own group.

Proof: Suppose $D M U_{o}$ belongs to $G_{k}$. To calculate the efficiency score of $D M U_{o}$ in its own group, using model (8), we set $\mu^{k}=1$ and for each $j \neq k, \mu_{j}=0$. With these values to $\mu^{j}$, model (8) reduces to the following form:

$$
\begin{array}{ll}
z_{k}=\operatorname{Max} \sum_{i=1}^{m} \delta_{i}^{o}+\sum_{r=1}^{s} \rho_{r}^{0} & \\
\sum_{j \in j_{k}} \bar{\lambda}_{j k}^{k} x_{i j}^{k} \leq x-\delta_{i}^{o} d_{i o}^{x}, & i=1,2, \ldots, m \quad k=1,2, \ldots, K \\
\sum_{j \in j_{k}} \bar{\lambda}_{j k}^{k} y_{r j}^{k} \geq y+\rho_{r}^{o} d_{r o}^{y}, & r=1,2, \ldots, s \quad k=1,2, \ldots, K \\
0 \leq \bar{\lambda}_{j k}^{k} \geq \mu^{k} M, & k=1,2, \ldots, K \quad j \in j_{k} \\
\bar{\lambda}^{k}=\left(\bar{\lambda}_{1}^{k}, \ldots, \bar{\lambda}_{j k}^{k}\right) \in \bar{\Lambda}_{k}, & k=1,2, \ldots, K \\
\mu^{k} \in\{0,1\}, & k=1,2, \ldots, K \\
0 \leq \delta_{i} \geq, & i=1,2, \ldots, m \\
\rho_{r} \geq 0, & r=1,2, \ldots, s
\end{array}
$$

If $\left(\delta_{i}^{o *}, \rho_{r}^{o *}, \bar{\lambda}_{k}^{*}\right)$ is the optimal solution the model (9) with optimal value of $z^{*}$, then $\left(\delta_{i}^{o *}, \rho_{r}^{o *}, \bar{\lambda}_{k}^{*}\right.$, $\left.\mu^{k}=1\right)$ is a feasible solution to model (8) and this completes the proof.

Theorem 2 shows that a DMU may be efficient in its own group, but, when we come to evaluate it with whole group, it may be inefficient.

\section{The transfer between the groups}

In this section, the effect of transferring a specific DMU from its current group to another one is considered. Suppose we are interested to transfer a specific $D M U_{o} \in G_{A}$ from its current group to another group $G_{B}$. Clearly, this transmission may affect on the performance of $D M U_{o}$ and DMUs in $G_{B}$. The impact of the presence or absence of a DMU in a technology is shown in Figure 1. After including $D M U_{o}$ to $G_{B}$, the performance of DMUs in $G_{B}$ can easily be calculated by a classic DEA model. However, to determine the best group among $G_{A}$ and $G_{B}$ to $D M U_{o}$, the following model is proposed. 


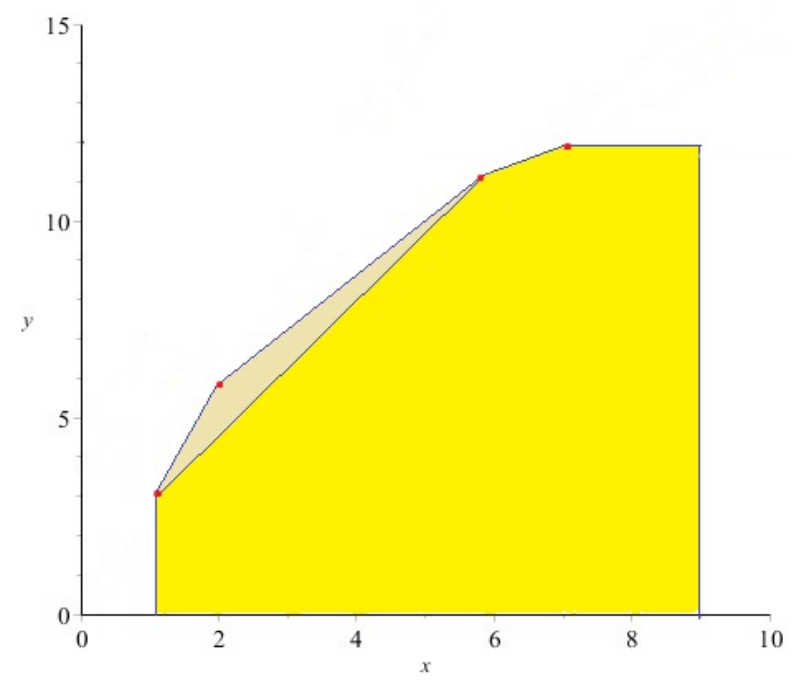

Figure 1: The impact of the presence and absence of a $D M U$

$$
\begin{aligned}
& \operatorname{Max} \sum_{i=1}^{m} \delta_{i}+\sum_{r=1}^{s} \rho_{r} \\
& \sum_{k \in A, B} \sum_{j \in j_{k}} \lambda_{j k}^{k} x_{i j}^{k}+d_{i}^{-}=\left(1-\delta_{i}\right) x_{i o}^{A}, \quad i=1,2, \ldots, m \\
& \sum_{k \in A, B} \sum_{j \in j_{k}} \lambda_{j k}^{k} y_{r j}^{k}-d_{r}^{+}=\left(1+\rho_{r}\right) y_{r o}^{A}, \quad r=1,2, \ldots, s \\
& \sum_{j \in j_{k}}^{K}=\mu^{k}, \quad k \in\{A, B\} \\
& \mu^{A}+\mu^{B}=1 \\
& \forall j \in j_{A}, \quad 0 \leq \lambda_{j}^{A} \leq \mu^{A} M \\
& \forall j \in j_{B}, \quad 0 \leq \lambda_{j}^{B} \leq \mu^{B} M \\
& \mu^{A}, \mu^{B} \in\{0,1\} \\
& \delta_{i}, \rho_{r}^{B} \geq 0, \quad i=1,2, \ldots, m \quad r=1,2, \ldots, s
\end{aligned}
$$

Note that in model given by eq.(10), we assumed $\left(d^{x}, d^{y}\right)=\left(x_{o}, x_{o}\right)$. In this model we assumed that groups $G_{A}$ and $G_{B}$ has two different technologies. In this model, if $\mu^{A}=1$ then $\mu^{B}=0$ and $G_{A}$ would be determined as the reference group to $D M U_{o}$.

At the end of this section, we illustrate the proposed approach with a small-scale example consisting eight DMUs in two groups. DMUs 1-4 belong to the first group and DMUs 5-8 are in second group. The DMUs use one input to produce one output. The data are listed in Table 1 and the two technology sets are depicted in Figure 2. The first three columns show the input and output data. The technical efficiency of the DMUs in their group is given in the fourth column of Table 1. As we can see, DMUs 1, 5 and 7 are efficient in their groups. 


\begin{tabular}{|l|cc|cc|ccc|}
\hline DMUs & input & output & DMUs TE in groups & meta frontier TE & $\mu^{1}$ & $\mu^{2}$ & group \\
\hline DMU1 & 1 & 2 & 0.000 & 0.500 & 0 & 1 & \\
DMU2 & 2 & 1 & 3.000 & 3.333 & 0 & 1 & group 1 \\
DMU3 & 2 & 3 & 0.333 & 0.500 & 0 & 1 & \\
DMU4 & 4 & 3 & 1.667 & 1.667 & 1 & 0 & \\
\hline DMU5 & 1 & 3 & 0.000 & 0.000 & 0 & 1 & \\
DMU6 & 2 & 4 & 0.125 & 0.125 & 0 & 1 & group 2 \\
DMU7 & 4 & 7 & 0.000 & 0.143 & 1 & 0 & \\
DMU8 & 5 & 1 & 6.200 & 9.000 & 1 & 0 & \\
\hline
\end{tabular}

Table 1: Data and results for eight DMUs

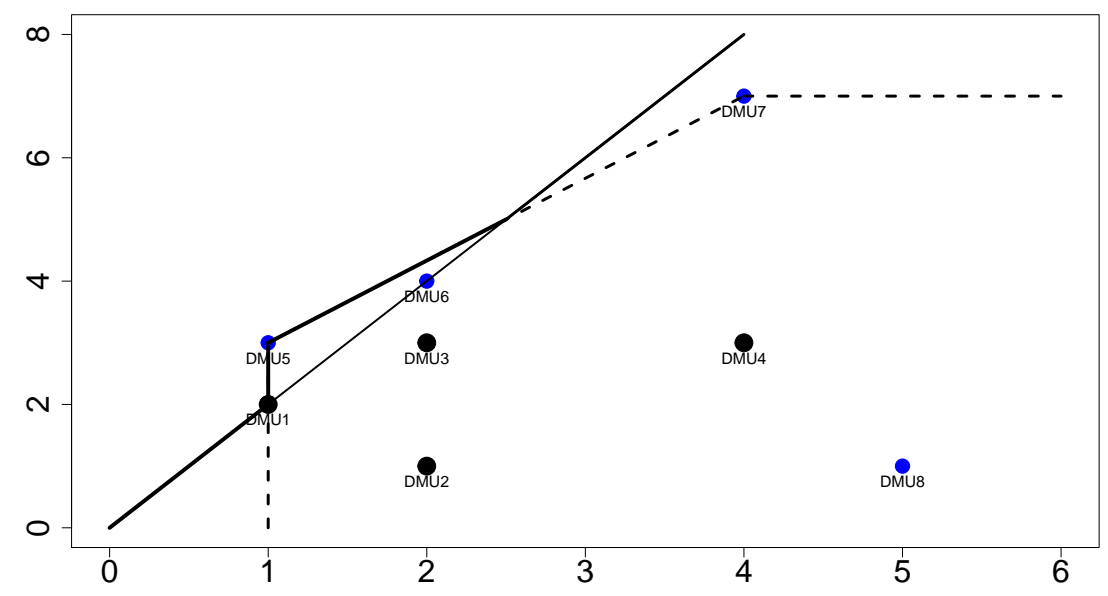

Figure 2: The meta-frontier

We applied our proposed model (8) to this data set. The results are given in columns 5 , 6 and 7 . As the results show, only one unit \#5 prevail as efficient. It is interesting to note that DMUs \#1 and \#7 that were efficient in their group are now in-efficient. Moreover, the reference group to $D M U_{1} \in G_{1}$ is $G_{2}$ and the reference group to $D M U_{7} \in G_{2}$ is $G_{1}$. Finally, comparing the columns 4 and 5 , we can see that the new inefficiency score of all DMUs are greater than or equal to the previous one.

\section{An application to Iranian banking industry}

In this section, we apply the model developed in this paper for the purpose of evaluating the technical efficiency in a major Iranian bank. In performance analysis in banking sector, DEA is one of the most frequently used tool to evaluate the relative efficiency of bank branches. In the last two decades, a lot of research studies have been conducted to evaluate the bank efficiency using DEA. In what follows, we briefly review some of these works.

Silva et al. [14] made a comparison of DEA and stochastic frontier approach (SFA) to investigate the efficiency of Chinese local commercial and rural banks. [13] made a survey to 80 published DEA applications in the bank branches of 24 countries. They studied the design of DEA models in these papers. [4] reviewed the recent developments related to empirical 
methodological advances of bank performance analysis. They selected 103 researches and analyzed them based on their efficiency measures, input-output approaches and methodologies. Ahn and Le [1] investigated the application of DEA in measuring bank efficiency They discussed some pitfalls which have been occurred in choosing inputs, outputs, weights and other factors. Recently [12] make a survey to the application of DEA in the banking industry.

In what follows, we focus on the performance evaluation of 92 bank branches of an Iranian commercial bank. According to Iranian banking law, based on the operation volume, the branches are divided into six degrees 1-6. Since the branches in the third, fourth and fifth degrees are the most homogeneous branches, our performance evaluation is focused on these branches. We collect the data from operations in 2017. Seven variables have been used as inputs and outputs in this analysis.

Inputs include number of staff, non-operational costs and assets. Outputs are deposits, profit, charges and loans (all monetary variables are stated in 10 thousands of current Iranian Rials). The data set for these 92 branches are given in Table 2. Note that the first thirty branches belong to $G_{4}$ (branches with degree 4), the second 36 branches belong to $G_{3}$ and the last 26 branches belong to $G_{5}$. After calculating the Pearson correlation coefficients of each two variables, it has been indicated that we cannot ignore none of these variables.

We first evaluated the branches in their own groups and we saw that of 92 branches, 45 branches are efficient. Now, we make the conditions more rigorous and model 7 has been used to this data set. The relative efficiency of the branches along with the projection points and the reference groups are given in Table 3. As the second column of Table 3 shows, the number of efficient branches has reduced from 45 to 28 . The membership values $\mu^{1}, \mu^{2}$ and $\mu^{3}$ are given in columns 3-5, respectively.

Consider branch 1. This branch is inefficient in both cases and $\mu^{1}=1$ and $\mu^{2}=\mu^{3}=0$ means that its frontier projection point belongs to the boundary of technology $G_{4}$. In other words, the most solid group to this branch is $G_{4}$.

Now consider branch 2. This branch was efficient in its original group, while, when it has been compared to $G_{5}$, it prevail as inefficient. This means that the most serious competitors to this branch belong to $G_{5}$. As we saw in this application, of 92 branches, 17 branches are efficient in their own group and when we extend their evaluation context, they would be determined as inefficient. This means that these 17 branches were overestimated in their own group. The data for this analysis are derived from operations during the first 6 months of 2018 . 


\begin{tabular}{|c|c|c|c|c|c|c|c|c|c|}
\hline J & $k$ & staff & costs & assets & deposits & profit & charge & loans & $\mathrm{TE}$ \\
\hline 1 & 4 & 17.67 & 32.15 & 3204.07 & 295207.1 & 60272.84 & 8091.01 & 114515.8 & 3.06 \\
\hline 2 & 4 & 13.26 & 27.77 & 1693.66 & 273151.3 & 55083.47 & 1064.62 & 231965.2 & 0.00 \\
\hline 3 & 4 & 11.96 & 29.26 & 2241.59 & 329613.6 & 75425.89 & 8929.29 & 142206.2 & 0.00 \\
\hline 4 & 4 & 10.36 & 15.88 & 3380.67 & 257394.6 & 77502.37 & 8232.96 & 195372.2 & 0.00 \\
\hline 5 & 4 & 11.31 & 12.14 & 3748.06 & 342367.3 & 56423.84 & 7791.50 & 217331.8 & 0.00 \\
\hline 6 & 4 & 10.14 & 21.08 & 2738.06 & 330547.5 & 41158.48 & 8775.26 & 227504.5 & 0.00 \\
\hline 7 & 4 & 12.28 & 21.38 & 3369.30 & 261761.8 & 74911.95 & 9393.61 & 299683.4 & 0.00 \\
\hline 8 & 4 & 14.43 & 29.60 & 2725.98 & 297084.0 & 68539.69 & 7829.84 & 115560.6 & 2.58 \\
\hline 9 & 4 & 12.84 & 31.31 & 2348.56 & 285941.6 & 53048.49 & 8823.33 & 279750.8 & 1.28 \\
\hline 10 & 4 & 13.41 & 12.80 & 2689.27 & 297157.2 & 41075.47 & 8215.23 & 262026.7 & 0.00 \\
\hline 11 & 4 & 17.81 & 15.77 & 2469.29 & 275714.9 & 77014.07 & 1026.15 & 134198.1 & 0.00 \\
\hline 12 & 4 & 16.45 & 13.71 & 2642.67 & 320751.1 & 48507.34 & 8206.47 & 231765.0 & 0.00 \\
\hline 13 & 4 & 17.93 & 14.54 & 3571.45 & 344216.7 & 74733.73 & 8204.02 & 242432.0 & 0.00 \\
\hline 14 & 4 & 13.73 & 25.34 & 2547.62 & 325392.9 & 67022.31 & 8121.02 & 222130.3 & 0.00 \\
\hline 15 & 4 & 12.40 & 31.41 & 1762.66 & 298896.8 & 67658.31 & 1057.76 & 124088.0 & 0.00 \\
\hline 16 & 4 & 16.21 & 33.40 & 2322.38 & 299345.4 & 59837.03 & 9112.26 & 131226.4 & 2.26 \\
\hline 17 & 4 & 12.87 & 29.05 & 2785.44 & 327187.4 & 58887.30 & 8804.32 & 151428.0 & 1.05 \\
\hline 18 & 4 & 13.90 & 26.79 & 2590.02 & 265506.5 & 49964.90 & 7941.57 & 231802.6 & 1.84 \\
\hline 19 & 4 & 10.33 & 34.67 & 3365.09 & 280549.0 & 53860.29 & 1043.09 & 167049.9 & 0.00 \\
\hline 20 & 4 & 11.85 & 20.46 & 1889.08 & 330483.4 & 64912.87 & 7682.65 & 177879.6 & 0.00 \\
\hline 21 & 4 & 17.41 & 26.84 & 2422.96 & 258813.7 & 57878.96 & 1075.00 & 233516.8 & 1.39 \\
\hline 22 & 4 & 10.80 & 19.69 & 2213.23 & 287846.0 & 76741.84 & 1091.55 & 280376.4 & 0.00 \\
\hline 23 & 4 & 12.05 & 24.36 & 2336.70 & 311613.8 & 76902.98 & 8860.29 & 304056.5 & 0.00 \\
\hline 24 & 4 & 15.44 & 23.75 & 2042.31 & 323177.3 & 77361.98 & 9258.39 & 239353.9 & 0.00 \\
\hline 25 & 4 & 16.05 & 24.64 & 2417.98 & 301945.6 & 59873.65 & 8063.77 & 195359.7 & 1.42 \\
\hline 26 & 4 & 14.42 & 18.27 & 2201.93 & 312154.0 & 51484.73 & 1046.29 & 148193.5 & 0.00 \\
\hline 27 & 4 & 10.48 & 23.00 & 2061.26 & 262576.7 & 52080.45 & 8165.35 & 125564.4 & 0.00 \\
\hline 28 & 4 & 16.09 & 11.04 & 2432.86 & 306486.7 & 60224.01 & 7982.80 & 127022.2 & 0.00 \\
\hline 29 & 4 & 17.89 & 27.82 & 3230.53 & 259216.6 & 61998.96 & 1063.56 & 175721.0 & 1.97 \\
\hline 30 & 4 & 15.37 & 33.23 & 2444.65 & 343557.5 & 42072.82 & 9312.11 & 120647.0 & 0.00 \\
\hline 31 & 3 & 10.81 & 16.38 & 1899.61 & 423833.4 & 43752.56 & 9734.46 & 149745.0 & 0.00 \\
\hline 32 & 3 & 17.08 & 25.51 & 3084.53 & 352108.8 & 63259.99 & 8537.17 & 287840.2 & 2.49 \\
\hline 33 & 3 & 10.12 & 29.22 & 1784.00 & 404359.6 & 69058.50 & 9187.99 & 306496.5 & 0.00 \\
\hline 34 & 3 & 10.26 & 21.29 & 3043.68 & 377625.4 & 78507.03 & 9069.75 & 297462.4 & 0.00 \\
\hline 35 & 3 & 11.76 & 22.08 & 2202.98 & 413313.7 & 71972.41 & 1025.22 & 129124.3 & 0.00 \\
\hline 36 & 3 & 12.86 & 26.57 & 1939.69 & 396614.0 & 43814.81 & 7719.83 & 161312.9 & 2.10 \\
\hline 37 & 3 & 12.43 & 30.42 & 3165.39 & 446841.3 & 40048.83 & 1047.89 & 129437.1 & 3.28 \\
\hline 38 & 3 & 12.05 & 20.87 & 3575.59 & 387431.0 & 70326.85 & 8329.52 & 151071.4 & 1.91 \\
\hline 39 & 3 & 17.61 & 34.65 & 2530.99 & 399086.0 & 71580.55 & 8498.93 & 203561.7 & 2.46 \\
\hline 40 & 3 & 10.43 & 29.84 & 1693.94 & 444012.3 & 51197.85 & 8901.52 & 167444.4 & 0.00 \\
\hline 41 & 3 & 16.53 & 22.97 & 3246.32 & 352017.3 & 79128.39 & 1088.40 & 106213.7 & 4.23 \\
\hline 42 & 3 & 12.81 & 26.35 & 3648.67 & 427962.6 & 76403.70 & 8838.28 & 244440.3 & 1.24 \\
\hline 43 & 3 & 11.59 & 12.78 & 3218.10 & 352969.5 & 78353.22 & 1093.12 & 156264.1 & 0.00 \\
\hline 44 & 3 & 10.51 & 28.77 & 2405.27 & 381479.8 & 65797.91 & 1052.70 & 187689.4 & 0.00 \\
\hline 45 & 3 & 12.99 & 18.66 & 1622.84 & 437978.8 & 50560.63 & 9498.72 & 195722.5 & 0.00 \\
\hline 46 & 4 & 17.89 & 30.04 & 2512.88 & 381827.8 & 40636.01 & 9158.30 & 308273.3 & 2.45 \\
\hline
\end{tabular}

Table 2a: Data for 92 bank branches 


\begin{tabular}{|c|c|c|c|c|c|c|c|c|c|}
\hline$j$ & $k$ & staff & costs & assets & deposits & profit & charge & loans & TE \\
\hline 47 & 3 & 10.04 & 28.56 & 3783.93 & 381479.8 & 56359.14 & 8998.29 & 136913.4 & 2.02 \\
\hline 48 & 3 & 16.21 & 34.37 & 3383.34 & 375711.8 & 65141.15 & 8844.16 & 215016.9 & 3.01 \\
\hline 49 & 3 & 16.47 & 10.45 & 2069.40 & 373706.8 & 60574.36 & 10704.12 & 247537.2 & 0.00 \\
\hline 50 & 3 & 15.79 & 34.27 & 1724.34 & 356860.6 & 59265.72 & 9695.79 & 178305.0 & 1.11 \\
\hline 51 & 3 & 10.68 & 28.26 & 2355.09 & 412627.0 & 54349.80 & 8802.39 & 230820.3 & 0.00 \\
\hline 52 & 3 & 11.06 & 10.80 & 1814.25 & 351348.9 & 47168.19 & 1028.18 & 278862.4 & 0.00 \\
\hline 53 & 3 & 15.01 & 21.21 & 2606.02 & 445376.4 & 52157.35 & 1045.48 & 179431.1 & 2.11 \\
\hline 54 & 3 & 13.24 & 23.39 & 2608.13 & 377579.6 & 55129.86 & 9651.89 & 309931.2 & 1.11 \\
\hline 55 & 3 & 14.44 & 23.29 & 3165.95 & 384336.4 & 57035.43 & 10129.89 & 106495.3 & 3.79 \\
\hline 56 & 3 & 11.45 & 34.19 & 1639.54 & 404240.5 & 59484.24 & 10669.73 & 147011.0 & 0.00 \\
\hline 57 & 3 & 14.23 & 10.37 & 3664.32 & 357867.7 & 41782.28 & 1087.91 & 251272.2 & 0.00 \\
\hline 58 & 3 & 16.45 & 31.33 & 2090.32 & 435570.8 & 68117.31 & 10867.34 & 225877.9 & 1.06 \\
\hline 59 & 3 & 12.10 & 34.49 & 3546.04 & 403608.8 & 77946.71 & 10058.96 & 264760.7 & 1.12 \\
\hline 60 & 3 & 16.93 & 23.34 & 2231.13 & 442684.7 & 62267.53 & 9181.91 & 224657.9 & 1.40 \\
\hline 61 & 3 & 10.92 & 28.21 & 3383.97 & 389564.2 & 68957.18 & 8987.72 & 154581.1 & 1.73 \\
\hline 62 & 3 & 15.91 & 15.34 & 2524.32 & 424025.7 & 41972.72 & 10823.86 & 226753.7 & 1.80 \\
\hline 63 & 3 & 17.23 & 30.34 & 2599.43 & 391798.2 & 52660.30 & 7936.34 & 130231.6 & 4.18 \\
\hline 64 & 3 & 14.36 & 30.05 & 3186.94 & 362573.6 & 67826.78 & 9075.52 & 289316.7 & 1.75 \\
\hline 65 & 3 & 11.17 & 26.69 & 3202.31 & 447216.7 & 46954.56 & 7879.30 & 260062.3 & 0.00 \\
\hline 66 & 3 & 15.85 & 24.66 & 3272.08 & 362930.7 & 68493.30 & 7523.71 & 243652.0 & 2.55 \\
\hline 67 & 5 & 13.06 & 23.41 & 2741.43 & 242251.4 & 55098.12 & 9991.24 & 277798.9 & 0.00 \\
\hline 68 & 5 & 14.77 & 32.71 & 2092.07 & 187180.7 & 49996.64 & 9530.98 & 149857.6 & 2.23 \\
\hline 69 & 5 & 17.19 & 28.80 & 2003.28 & 2211 & 530 & 37 & & 2.30 \\
\hline 70 & 5 & 16.91 & 28.24 & 1969.87 & 193 & 44301.89 & 970 & 2965 & 1.03 \\
\hline 71 & 5 & 11.11 & 29.85 & & 157269.5 & 61463.06 & 10473.83 & 131520.4 & 0.00 \\
\hline 72 & 5 & 12.74 & 14.90 & 3107.27 & 150073.2 & 43285.01 & 10517.94 & 298019.2 & 0.00 \\
\hline 73 & 5 & 12.97 & 19.40 & 2235.27 & 197141.9 & 58374.58 & 10681.59 & 1081 & 0.00 \\
\hline 74 & 5 & 17.28 & 12.11 & 1927.68 & 235155.8 & 75436.87 & 9804.32 & 2673 & 0.00 \\
\hline 75 & 5 & 13.73 & 19.44 & 2726.76 & 230358.3 & 44647.36 & 7932.07 & 153617.7 & 1.82 \\
\hline 76 & 5 & 15.64 & 17.60 & 1668.88 & 208302.6 & 59763.79 & 9566.97 & 209523.9 & 0.00 \\
\hline 77 & 5 & 17.78 & 22.42 & 1975.91 & 159558.4 & 43877.07 & 10445.10 & 157421.5 & 2.22 \\
\hline 78 & 5 & 16.00 & 25.14 & 3540.29 & 206251.7 & 48806.42 & 8816.60 & 234348.9 & 2.27 \\
\hline 79 & 5 & 10.59 & 19.05 & 3589.63 & 222200.7 & 72793.97 & 9856.01 & 258304.2 & 0.00 \\
\hline 80 & 5 & 14.09 & 20.41 & 3381.16 & 166580.7 & 75825.07 & 8378.87 & 151503.0 & 1.92 \\
\hline 81 & 5 & 11.39 & 12.35 & 2664.92 & 192756.4 & 72448.50 & 10627.54 & 152754.3 & 0.00 \\
\hline 82 & 5 & 15.69 & 17.98 & 2350.17 & 181421.9 & 64041.26 & 9091.22 & 232866.1 & 0.00 \\
\hline 83 & 5 & 17.76 & 13.14 & 1999.42 & 226284.1 & 67583.85 & 9552.13 & 190705.0 & 0.00 \\
\hline 84 & 5 & 15.50 & 27.41 & 2996.86 & 162002.9 & 57709.28 & 10242.58 & 167982.1 & 2.47 \\
\hline 85 & 5 & 16.37 & 34.30 & 3560.99 & 185331.3 & 53069.25 & 7933.45 & 267851.3 & 2.78 \\
\hline 86 & 5 & 11.42 & 27.42 & 1891.96 & 243304.2 & 58447.83 & 8901.20 & 293020.4 & 0.00 \\
\hline 87 & 5 & 17.40 & 34.94 & 1623.47 & 233425.4 & 54676.96 & 8801.54 & 260487.7 & 0.00 \\
\hline 88 & 5 & 14.01 & 27.05 & 2287.70 & 195860.2 & 43471.79 & 10449.26 & 188271.3 & 1.71 \\
\hline 89 & 5 & 15.40 & 23.60 & 1518.53 & 159375.3 & 47613.76 & 9938.15 & 216762.4 & 0.00 \\
\hline 90 & 5 & 13.92 & 30.93 & 1907.54 & 227922.9 & 78413.04 & 7940.40 & 135899.8 & 0.00 \\
\hline 91 & 5 & 10.30 & 22.58 & 2964.57 & 157956.2 & 46695.76 & 7917.11 & 249132.5 & 1.05 \\
\hline 92 & 5 & 16.37 & 13.49 & 2193.43 & 239632.9 & 46816.61 & 8914.98 & 305852.1 & 0.00 \\
\hline
\end{tabular}

Table 2b: Data for 92 bank branches (continued) 


\begin{tabular}{|c|c|c|c|c|c|c|c|c|c|c|c|c|}
\hline$j$ & $k$ & staff & costs & assets & deposits & profit & charge & loans & $\mathrm{TE}$ & $\mu^{1}$ & $\mu^{2}$ & $\mu^{3}$ \\
\hline 1 & 4 & 17.7 & 32.1 & 2994.4 & 625257.1 & 95321.9 & 16259.5 & 485136.0 & 6.01 & 1 & 0 & 0 \\
\hline 2 & 4 & 13.3 & 27.8 & 1693.7 & 273151.3 & 55083.5 & 10646.2 & 231965.2 & 0.00 & 0 & 1 & 0 \\
\hline 3 & 4 & 12.0 & 29.3 & 2131.5 & 455560.2 & 75425.9 & 10907.7 & 348715.8 & 2.11 & 1 & 0 & 0 \\
\hline 4 & 4 & 10.4 & 15.9 & 3380.7 & 257394.6 & 77502.4 & 8233.0 & 195372.2 & 0.00 & 0 & 1 & 0 \\
\hline 5 & 4 & 11.3 & 12.1 & 2212.1 & 360163.0 & 56423.8 & 10516.1 & 261370.6 & 1.01 & 1 & 0 & 0 \\
\hline 6 & 4 & 10.1 & 21.1 & 2738.1 & 372588.1 & 73509.4 & 90328.0 & 292174.0 & 1.23 & 1 & 0 & 0 \\
\hline 7 & 4 & 12.3 & 21.4 & 2727.6 & 432133.0 & 74912.0 & 11139.2 & 339090.5 & 1.16 & 1 & 0 & 0 \\
\hline 8 & 4 & 14.4 & 29.6 & 2467.0 & 524995.4 & 82331.9 & 13241.4 & 405099.9 & 4.26 & 1 & 0 & 0 \\
\hline 9 & 4 & 12.8 & 31.3 & 2228.0 & 488594.6 & 79927.5 & 11731.7 & 373782.6 & 1.93 & 1 & 0 & 0 \\
\hline 10 & 4 & 13.1 & 12.8 & 2150.3 & 416428.1 & 55905.0 & 12186.2 & 330515.2 & 1.73 & 1 & 0 & 0 \\
\hline 11 & 4 & 17.8 & 15.8 & 2469.3 & 275714.9 & 77014.1 & 10261.5 & 134198.1 & 0.00 & 0 & 1 & 0 \\
\hline 12 & 4 & 14.0 & 13.7 & 2303.4 & 446071.4 & 59884.6 & 13053.7 & 354042.8 & 2.02 & 1 & 0 & 0 \\
\hline 13 & 4 & 17.9 & 14.5 & 2754.5 & 483839.7 & 74733.7 & 14105.7 & 340724.7 & 1.76 & 1 & 0 & 0 \\
\hline 14 & 4 & 13.7 & 25.3 & 2329.5 & 487465.0 & 74561.7 & 12632.2 & 377982.4 & 1.95 & 1 & 0 & 0 \\
\hline 15 & 4 & 12.4 & 31.4 & 1762.7 & 298896.8 & 67658.3 & 10577.6 & 124088.0 & 0.00 & 0 & 1 & 0 \\
\hline 16 & 4 & 13.4 & 33.4 & 2322.4 & 511714.9 & 84247.0 & 12190.6 & 390944.8 & 3.61 & 1 & 0 & 0 \\
\hline 17 & 4 & 12.9 & 29.0 & 2216.9 & 479509.4 & 76965.1 & 11777.9 & 368275.1 & 2.75 & 1 & 0 & 0 \\
\hline 18 & 4 & 13.9 & 26.8 & 2364.9 & 498249.2 & 76992.0 & 12771.9 & 385581.4 & 2.78 & 1 & 0 & 0 \\
\hline 19 & 4 & 10.3 & 34.7 & 3365.1 & 280549.0 & 53860.3 & 10430.9 & 167049.9 & 0.00 & 0 & 1 & 0 \\
\hline 20 & 4 & 11.8 & 20.5 & 1889.1 & 330483.4 & 64912.9 & 7682.7 & 177879.6 & 0.00 & 0 & 1 & 0 \\
\hline 21 & 4 & 14.3 & 26.8 & 2423.0 & 508554.7 & 78141.2 & 13115.4 & 393989.8 & 2.40 & 1 & 0 & 0 \\
\hline 22 & 4 & 10.8 & 19.7 & 2213.2 & 287846.0 & 76741.8 & 10915.5 & 280376.4 & 0.00 & 0 & 1 & 0 \\
\hline 23 & 4 & 12.1 & 24.4 & 2336.7 & 311613.8 & 76903.0 & 8860.3 & 304056.5 & 0.00 & 0 & 1 & 0 \\
\hline 24 & 4 & 15.4 & 23.7 & 2042.3 & 323177.3 & 77362.0 & 9258.4 & 239353.9 & 0.00 & 0 & 1 & 0 \\
\hline 25 & 4 & 14.7 & 14.4 & 2418.0 & 468266.9 & 62864.3 & 13703.2 & 371659.1 & 2.70 & 1 & 0 & 0 \\
\hline 26 & 4 & 13.4 & 13.1 & 2201.9 & 426426.0 & 57247.2 & 12478.8 & 338450.4 & 2.31 & 1 & 0 & 0 \\
\hline 27 & 4 & 10.5 & 23.0 & 1800.9 & 387645.2 & 61799.6 & 9596.8 & 298132.2 & 2.34 & 1 & 0 & 0 \\
\hline 28 & 4 & 15.9 & 11.0 & 2106.8 & 386239.2 & 60224.0 & 11116.2 & 267071.2 & 1.90 & 1 & 0 & 0 \\
\hline 29 & 4 & 17.9 & 27.8 & 3001.7 & 612814.4 & 90175.0 & 16517.9 & 478658.4 & 4.17 & 1 & 0 & 0 \\
\hline 30 & 4 & 14.1 & 33.2 & 2444.7 & 532545.9 & 86328.6 & 12928.2 & 408176.9 & 4.45 & 1 & 0 & 0 \\
\hline 31 & 3 & 10.8 & 16.4 & 1899.6 & 423833.4 & 43752.6 & 9734.5 & 149745.0 & 0.00 & 1 & 0 & 0 \\
\hline 32 & 3 & 17.1 & 16.7 & 2801.7 & 542584.2 & 72841.3 & 15878.0 & 430644.1 & 2.49 & 1 & 0 & 0 \\
\hline 33 & 3 & 10.1 & 29.2 & 1784.0 & 404359.6 & 69058.5 & 9188.0 & 306496.5 & 0.00 & 1 & 0 & 0 \\
\hline 34 & 3 & 10.3 & 21.3 & 3043.7 & 377625.4 & 78507.0 & 9069.8 & 297462.4 & 0.00 & 1 & 0 & 0 \\
\hline 35 & 3 & 11.8 & 22.1 & 2203.0 & 413313.7 & 71972.4 & 10252.2 & 129124.3 & 0.00 & 1 & 0 & 0 \\
\hline 36 & 3 & 11.2 & 26.6 & 1939.7 & 423193.7 & 68747.0 & 10247.6 & 324220.7 & 2.10 & 1 & 0 & 0 \\
\hline 37 & 3 & 12.4 & 30.4 & 3003.9 & 475569.2 & 90433.7 & 11127.7 & 367855.0 & 3.28 & 1 & 0 & 0 \\
\hline 38 & 3 & 12.1 & 20.9 & 2477.2 & 423030.3 & 70326.9 & 10984.3 & 331074.5 & 1.91 & 1 & 0 & 0 \\
\hline 39 & 3 & 14.6 & 34.6 & 2531.0 & 552143.6 & 89681.7 & 13372.4 & 423025.6 & 2.46 & 1 & 0 & 0 \\
\hline 40 & 3 & 10.4 & 29.8 & 1693.9 & 444012.3 & 51197.9 & 8901.5 & 167444.1 & 0.00 & 1 & 0 & 0 \\
\hline 41 & 3 & 16.5 & 23.0 & 2756.2 & 554553.1 & 79662.6 & 15294.7 & 435047.0 & 4.23 & 1 & 0 & 0 \\
\hline 42 & 3 & 12.8 & 26.4 & 2401.5 & 466981.3 & 76403.7 & 11700.7 & 361310.5 & 1.24 & 1 & 0 & 0 \\
\hline 43 & 3 & 11.6 & 12.8 & 3218.1 & 352969.5 & 78353.2 & 10931.2 & 156264.1 & 0.00 & 1 & 0 & 0 \\
\hline 44 & 3 & 10.5 & 28.8 & 2405.3 & 381479.8 & 65797.9 & 10527.0 & 187689.4 & 0.00 & 1 & 0 & 0 \\
\hline 45 & 3 & 13.0 & 18.7 & 1622.8 & 437978.8 & 50560.6 & 9498.7 & 195722.5 & 0.00 & 1 & 0 & 0 \\
\hline 46 & 3 & 14.7 & 30.0 & 2512.9 & 534406.9 & 83729.6 & 13492.8 & 412438.5 & 2.45 & 1 & 0 & 0 \\
\hline
\end{tabular}

Table 3a: Meta TE results and the projection points 


\begin{tabular}{|c|c|c|c|c|c|c|c|c|c|c|c|c|}
\hline$j$ & $k$ & staff & costs & assets & deposits & profit & charge & loans & $\mathrm{TE}$ & $\mu^{1}$ & $\mu^{2}$ & $\mu^{3}$ \\
\hline 47 & 3 & 10.0 & 28.6 & 1834.9 & 399622.4 & 68979.9 & 9105.9 & 303491.1 & 2.02 & 1 & 0 & 0 \\
\hline 48 & 3 & 16.2 & 34.4 & 2778.0 & 594449.5 & 93973.7 & 14858.9 & 457959.6 & 3.01 & 1 & 0 & 0 \\
\hline 49 & 3 & 16.5 & 10.5 & 2069.4 & 373706.8 & 60574.4 & 10704.1 & 247537.2 & 0.00 & 1 & 0 & 0 \\
\hline 50 & 3 & 10.5 & 24.7 & 1724.3 & 382041.5 & 59265.7 & 9695.8 & 253669.4 & 1.11 & 1 & 0 & 0 \\
\hline 51 & 3 & 10.7 & 28.3 & 1866.9 & 415829.4 & 69447.2 & 9729.7 & 316725.1 & 0.00 & 1 & 0 & 0 \\
\hline 52 & 3 & 11.1 & 10.8 & 1814.3 & 351348.9 & 47168.2 & 10281.8 & 278862.4 & 0.00 & 1 & 0 & 0 \\
\hline 53 & 3 & 15.0 & 14.7 & 2463.0 & 476979.3 & 64033.9 & 13958.2 & 378574.1 & 2.11 & 1 & 0 & 0 \\
\hline 54 & 3 & 13.2 & 12.9 & 2172.0 & 420623.6 & 56468.2 & 12309.0 & 333845.1 & 1.11 & 1 & 0 & 0 \\
\hline 55 & 3 & 14.4 & 23.3 & 2428.4 & 498238.8 & 73905.0 & 13324.0 & 388589.1 & 3.79 & 1 & 0 & 0 \\
\hline 56 & 3 & 11.4 & 34.2 & 1639.5 & 404240.5 & 59484.2 & 10669.7 & 147011.0 & 0.00 & 1 & 0 & 0 \\
\hline 57 & 3 & 14.2 & 10.4 & 3664.3 & 357867.7 & 41782.3 & 10879.1 & 251272.2 & 0.00 & 1 & 0 & 0 \\
\hline 58 & 3 & 12.3 & 23.7 & 2090.3 & 440563.9 & 68117.3 & 11286.2 & 340902.1 & 1.06 & 1 & 0 & 0 \\
\hline 59 & 3 & 12.1 & 34.5 & 2130.6 & 481659.9 & 81989.4 & 10992.9 & 365353.3 & 1.12 & 1 & 0 & 0 \\
\hline 60 & 3 & 13.5 & 16.8 & 2231.1 & 443142.9 & 62267.5 & 12471.0 & 349005.1 & 1.40 & 1 & 0 & 0 \\
\hline 61 & 3 & 10.9 & 28.2 & 1904.2 & 422196.8 & 70087.5 & 9954.5 & 321988.5 & 1.73 & 1 & 0 & 0 \\
\hline 62 & 3 & 15.4 & 15.0 & 2524.3 & 488861.0 & 65629.0 & 14305.9 & 388004.5 & 1.80 & 1 & 0 & 0 \\
\hline 63 & 3 & 15.2 & 30.3 & 2599.4 & 550464.6 & 85708.9 & 13994.3 & 425355.8 & 4.18 & 1 & 0 & 0 \\
\hline 64 & 3 & 14.4 & 30.1 & 2459.0 & 525004.7 & 82726.9 & 13171.1 & 404722.3 & 1.75 & 1 & 0 & 0 \\
\hline 65 & 3 & 11.2 & 26.7 & 3202.3 & 447216.7 & 46954.6 & 7879.3 & 260062.3 & 0.00 & 1 & 0 & 0 \\
\hline 66 & 3 & 15.9 & 16.1 & 2605.2 & 506499.6 & 68493.3 & 14733.2 & 401519.0 & 2.55 & 1 & 0 & 0 \\
\hline 67 & 5 & 13.1 & 23.4 & 2210.6 & 460570.7 & 69978.6 & 12019.3 & 357587.3 & 1.86 & 0 & 1 & 0 \\
\hline 68 & 5 & 11.9 & 32.7 & 2092.1 & 469266.8 & 79088.5 & 10851.8 & 356726.1 & 3.80 & 1 & 0 & 0 \\
\hline 69 & 5 & 11.5 & 28.8 & 2003.3 & 441 & 72664.5 & 10515.9 & 337218.8 & 3.40 & 0 & 1 & 0 \\
\hline 70 & 5 & 13.1 & 28.2 & 1969.9 & 481641.3 & 70041.2 & 10720.9 & 296580.2 & 2.40 & 1 & 0 & 0 \\
\hline 71 & 5 & 11.1 & 29.8 & 2154.5 & 422526.5 & 71405.4 & 10473.8 & 283336.1 & 3.07 & 0 & 1 & 0 \\
\hline 72 & 5 & 12.7 & 14.9 & 2387.1 & 416182.5 & 61925.2 & 11747.9 & 329829.3 & 2.66 & 1 & 0 & 0 \\
\hline 73 & 5 & 13.0 & 19.4 & 2172.0 & 441119.9 & 64362.9 & 11988.0 & 345085.9 & 3.68 & 1 & 0 & 0 \\
\hline 74 & 5 & 17.3 & 12.1 & 1927.7 & 235155.8 & 75436.9 & 9804.3 & 267357.1 & 0.00 & 0 & 0 & 1 \\
\hline 75 & 5 & 13.7 & 19.4 & 2291.1 & 462079.7 & 66646.3 & 12696.3 & 362239.9 & 3.62 & 1 & 0 & 0 \\
\hline 76 & 5 & 15.6 & 17.6 & 1668.9 & 208302.6 & 59763.8 & 9567.0 & 209523.9 & 0.00 & 0 & 0 & 1 \\
\hline 77 & 5 & 11.6 & 2.2 & 1975.9 & 4164 & 64370.4 & 10669.2 & 322216.4 & 3.49 & 1 & 0 & 0 \\
\hline 78 & 5 & 16.0 & 25.1 & 3540.3 & 5514 & 94027.6 & 14544.3 & 4346 & 4.10 & 1 & 0 & 0 \\
\hline 79 & 5 & 10.6 & 19.1 & 3589.6 & 2222 & 72794.0 & 9856.0 & 25830 & 0.00 & 0 & 1 & 0 \\
\hline 80 & 5 & 14.1 & 20.4 & 2801.0 & 477 & 75825.1 & 12907.0 & 376044.2 & 4.06 & 1 & 0 & 0 \\
\hline 81 & 5 & 11.4 & 12.4 & 2664.9 & 192756.4 & 72448.5 & 10627.5 & 152754.3 & 0.00 & 0 & 0 & 1 \\
\hline 82 & 5 & 15.4 & 17.1 & 2350.2 & 498588.5 & 64041.3 & 13425.0 & 342366.8 & 2.76 & 0 & 0 & 1 \\
\hline 83 & 5 & 17.8 & 12.4 & 1981.2 & 241684.3 & 77531.2 & 10076.5 & 274779.6 & 0.00 & 0 & 0 & 1 \\
\hline 84 & 5 & 15.5 & 27.4 & 2621.2 & 545008.5 & 82548.2 & 14269.3 & 423398.8 & 4.83 & 0 & 1 & 0 \\
\hline 85 & 5 & 16.4 & 34.3 & 3561.0 & 600722.3 & 105918.2 & 14816.8 & 466736.0 & 4.85 & 1 & 0 & 0 \\
\hline 86 & 5 & 10.9 & 27.4 & 1892.0 & 417551.3 & 68893.3 & 9920.7 & 318859.1 & 1.15 & 1 & 0 & 0 \\
\hline 87 & 5 & 95.9 & 20.4 & 1623.5 & 349172.6 & 54677.0 & 8801.5 & 260487.7 & 1.36 & 1 & 0 & 0 \\
\hline 88 & 5 & 13.4 & 27.0 & 2287.7 & 485561.8 & 75857.9 & 12298.7 & 374955.2 & 3.44 & 1 & 0 & 0 \\
\hline 89 & 5 & 15.4 & 23.6 & 1518.5 & 159375.3 & 47613.8 & 9938.2 & 216762.4 & 0.00 & 0 & 0 & 1 \\
\hline 90 & 5 & 13.9 & 30.9 & 1907.5 & 227922.9 & 78413.0 & 7940.4 & 135899.8 & 0.00 & 0 & 0 & 1 \\
\hline 91 & 5 & 10.3 & 22.6 & 1771.0 & 381080.0 & 60724.5 & 9439.3 & 293110.8 & 2.48 & 1 & 0 & 0 \\
\hline 92 & 5 & 13.4 & 13.1 & 2193.4 & 424781.2 & 57026.4 & 12430.7 & 337144.9 & 1.70 & 1 & 0 & 0 \\
\hline
\end{tabular}

Table 3b: Meta TE results and the projection points (continued) 


\section{Conclusion}

A main assumption in performance measurement using tools such as DEA is that all firms are in a single technology set. However, in many real applications, we confront cases in which different groups of firms perform in different but similar environments. The differences in the nature of the DMUs and the region that they do business have led to different technology sets and in this case, we confront groups of DMUs that technologically are different but, this difference is not a significant difference. So, a DMU can be transferred between the groups to select best reference group.

In this paper, we have developed a DEA based procedure and a directional distance function model to evaluate DMUs in different groups. The proposed model has been used to evaluate the relative efficiency of 92 bank branches of an Iranian major bank. As the results showed, we saw that when we extended the evaluation context, the efficiency classification of the DMUs may be altered. A DMU may be efficient in its current group and when we assess this unit in a larger group, it may be inefficient. This shows the impact and importance of group efficiency analysis.

\section{References}

[1] Ahn, H., and Le, M. H. (2014). An insight into the specification of the input-output set for DEA-based bank efficiency measurement. Management Review Quarterly, 64(1), 3-37. doi: 10.1007/s11301-013-0098-9

[2] Banker, R. D., Charnes, A. and Cooper, W. W. (1984). Some Models for Estimating Technical and Scale Inefficiencies in Data Envelopment Analysis. Management Science, 30(9), 1078-1092. doi: $10.1287 / \mathrm{mnsc} .30 .9 .1078$

[3] Battese, G. E., Rao, D. S. P. and O'Donnell, C. J. (2004). A Metafrontier Production Function for Estimation of Technical Efficiencies and Technology Gaps for Firms Operating Under Different Technologies. Journal of Productivity Analysis, 21(1), 91-103. doi: 10.1023/b:prod.0000012454.06094.29

[4] Bhatia, V., Basu, S., Mitra, S. K. and Dash, P. (2018). A review of bank efficiency and productivity. OPSEARCH, 55(3-4), 557-600. doi: 10.1007/s12597-018-0332-2

[5] Charnes, A., Cooper, W. W. and Rhodes, E. (1978). Measuring the efficiency of decision making units. European Journal of Operational Research, 2(6), 429-444. doi: 10.1016/0377-2217(78)90138

[6] Emrouznejad, A., Parker, B. R. and Tavares, G. (2008). Evaluation of research in efficiency and productivity: A survey and analysis of the first 30 years of scholarly literature in DEA. SocioEconomic Planning Sciences, 42(3), 151-157. doi: 10.1016/j.seps.2007.07.002

[7] Farrell, M. J. (1957). The Measurement of Productive Efficiency. Journal of the Royal Statistical Society, Series A (General), 120(3), 253-290. doi: 10.2307/2343100

[8] Hayami, Y. and Ruttan, V. W. (1971). Agricultural development: An international perspective. Baltimore: Johns Hopkins University Press.

[9] Lovell, K. C. A. and Pastor, J. T. (1997). Target setting: An application to a bank branch network. European Journal of Operational Research, 98(2), 290-299. doi: 10.1016/s0377-2217(96)00348-7

[10] McMillan, M. L. and Chan, W. H. (2006). University Efficiency: A Comparison and Consolidation of Results from Stochastic and Non-stochastic Methods. Education Economics, 14(1), 1-30. doi: $10.1080 / 09645290500481857$

[11] O'Donnell, C. J., Rao, D. S. P. and Battese, G. E. (2008). Metafrontier frameworks for the study of firm-level efficiencies and technology ratios. Empirical Economics, 34(2), 231-255. doi: 10.1007/s00181-007-0119-4

[12] Paradi, J. C., Sherman, H. D. and Tam, F. K. (2018). Data Envelopment Analysis in the Financial Services Industry. International Book Series in Operations Research \& Management Science: Springer. doi: 10.1007/978-3-319-69725-3

[13] Paradi, J. C. and Zhu, H. (2013). A survey on bank branch efficiency and performance research with data envelopment analysis. Omega, 41(1), 61-79. doi: 10.1016/j.omega.2011.08.010 
[14] Silva, T. C., Tabak, B. M., Cajueiro, D. O. and Dias, M. V. B. (2017). A comparison of DEA and SFA using micro- and macro-level perspectives: Efficiency of Chinese local banks. Physica A: Statistical Mechanics and its Applications, 469, 216-223. doi: 10.1016/j.physa.2016.11.041

[15] Worthington, A. C. and Lee, B. L. (2008). Efficiency, technology and productivity change in Australian universities, 1998-2003. Economics of Education Review, 27(3), 285-298. doi: 10.1016/j.econedurev.2006.09.012 